Int. J. Dev. Biol. 52: 925-932 (2008)

doi: $10.1387 / \mathrm{ijdb} .072418 \mathrm{af}$

\title{
Integrins contribute to the establishment and maintenance of cell polarity in the follicular epithelium of the Drosophila ovary
}

\author{
ANA FERNÁNDEZ-MIÑÁN\#, LAURA COBREROS, ACAIMO GONZÁLEZ-REYES \\ and MARÍA D. MARTÍN-BERMUDO* \\ Centro Andaluz de Biología del Desarrollo, CSIC-Univ. Pablo de Olavide, Sevilla, Spain
}

\begin{abstract}
The generation of epithelial cell polarity is a key process during development. Although the induction and orientation of cell polarity by cell-cell and cell-extracellular matrix (ECM) interactions is well established, the molecular mechanisms by which signals from the ECM control cell polarity in developing epithelial tissues remain poorly understood. Here, we have used the follicular epithelium of the Drosophila ovary to investigate the role that integrins, the main cell-ECM receptors, play in the establishment of apicobasal polarity. Mature follicle cells have an apical side facing the germ line and a basal side in contact with a basement membrane. Our results show that integrins - presumably via interactions with the basement membrane - play a reinforcing role in follicle cell polarization, as they are required to establish and/or maintain follicle cell membrane asymmetry only when contact with the germ line is prevented. We suggest that the primary cue for polarization of the follicular epithelium is contact with the germline cells. In addition, while interfering with apical and lateral polarization cues leads to apoptosis, we show here that inhibition of contact with the basement membrane mediated by integrins does not affect cell survival. Finally, we provide evidence to suggest that integrins are required to orientate epithelial polarity in vivo.
\end{abstract}

KEY WORDS: integrin, apicobasal polarity, basement membrane, epithelial polarity

\section{Introduction}

Development and maintenance of epithelial polarity are crucial for all multicellular organisms. Within epithelial tissues, cell polarity regulates cellular morphology, intercellular signalling, asymmetric cell division, cell migration, cellular and tissular physiology as well as complex organ morphogenesis.

The molecular mechanism by which apicobasal polarity is generated and maintained has been intensively investigated and it has been shown to be highly conserved among cell types and organisms (for review see Margolis and Borg, 2005). Three major protein complexes are essential for epithelial polarization, the Par3/Par6/ atypical protein kinase C (aPKC) and the Crumbs/ PATJ/Stardust complexes act on the apical domain, and the Scribble/Disc Large/Lethal Giant Larvae complex on the lateral one. The current model proposes that the antagonistic activity of these apical and basolateral protein complexes leads to the polarized distribution of proteins and cell-cell junctions along the membrane of the cell, thus inducing cell polarity. In addition, cell polarization also requires cell-cell and cell-extracellular matrix (ECM) adhesion. Cell-cell adhesion mediated by cadherins appears to be an important step in promoting epithelial polarity by specifying the lateral membrane domain (Nelson, 2003). $\beta 1$ integrins, the main ECM receptors in the cell, play a key role in epithelial polarization as it was first recognized in kidney development (Sorokin et al., 1990). $\beta 1$ integrins have also been involved in the maintenance of apicobasal polarity of mammary epithelial cells, as cells isolated from transgenic glands where integrin function is compromised fail to polarize properly (Faraldo et al., $1998 b)$. In addition, members of the $\beta 1$ integrin family have also been involved in the regulation of epithelial polarity reversal in Madin-Darby canine kidney (MDCK) cells (Ojakian and

Abbreviations used in this paper: aPKC, atypical protein kinase C; ECM,
extracellular matrix; MDCK, Madin-Darbycanine kidney; SJ, septatejunction.

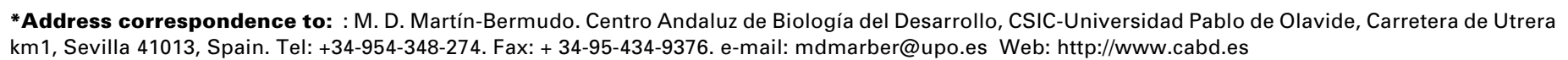

\#Current Address: EMBL, Heidelberg, Germany.

Published online: 20 August 2008

$0214-6282 / 2008 / \$ 35.00$

(C) UBC Press

Printed in Spain 
Schwimmer, 1994). However, the contribution of cell-ECM adhesion to epithelial polarization remains poorly understood and it has been mainly studied in cells in culture.

The follicular epithelium of the Drosophila ovary constitutes an ideal model system to analyse the mechanisms underlying formation and maintenance of apicobasal epithelial cell polarity in vivo. The Drosophila ovary is composed of ovarioles, each containing a germarium at their proximal end and progressively older egg chambers towards their distal end (Fig. 1A). Each egg chamber is composed of a cluster of 16 germline cells and a single layer of follicle epithelial cells (King, 1970, Spradling, 1993). Follicle cells develop from about two somatic stem cells per ovariole that are located in the boundary between regions $2 \mathrm{a}$ to $2 \mathrm{~b}$ of the germarium (Margolis and Spradling, 1995). These stem cells give rise to two populations of follicle cells, a population that covers the germ cell cluster in region 3 of the germarium, and a second population that undergoes cell intercalation and forms stacks connecting two consecutives egg chambers, the interfollicular stalk. The follicular epithelium displays features from both primary and secondary epithelia. On one hand, it is formed through a mesenchymal to epithelium transition, as secondary epithelia do (King, 1970). On the other hand, and similar to primary epithelia, the follicular epithelium presents a zonula adherens where $D E$-Cadherin, $\mathrm{D} \alpha$-Catenin and Armadillo ( $\beta$-Catenin) are localised (Mahowald, 1972; Tanentzapf et al., 2000). Protein complexes such as Crumbs-DPatj-Stardust, and the BazookaaPKC-DmPar6 complexes are found apically to the zonula adherens, in the marginal zone (for revirew see Tepass et al., 2001). The lateral and basal domains of the follicle cells are in contact with a laminin-rich extracellular matrix and contain $\beta P S$ integrins (Bateman et al., 2001, Brown et al., 2002). However, we have recently shown that, in addition to its normal basolateral localization, $\beta$ PS integrins are also found in the apical side of all follicle cells (Fernández-Miñán etal., 2007).

The analysis of follicular epithelium development has revealed that follicle cells use three different mechanisms to acquire an apicobasal polarity, each of them operating at the basal, apical and lateral membrane domains, respectively (Tanentzapf etal., 2000). First, during the mesenchyme to epithelium transition, contact with the basement membrane leads to an initial polarization by which the basal domain of follicle cells is established. Later, contact with germline cells in region 2 of the germarium (Fig. 1B) leads to the initial establishment of the apical and lateral domains. As a consequence, adherens junctions can be detected as early as Stage (S) 2. By S6, incipient septate adherens appear and by $\mathrm{S} 10$ they are completely formed (Muller, 2000). The polarization of follicle cells is essential, as it allows them to exert crucial functions throughout oogenesis. For example, interactions between the apical surface of the follicle cells and the germline cells participate in signalling events that lead to the establishment of the anterioposterior axis of the egg. In addition, follicle cells secrete the eggshell material from their apical membrane at later stages of oogenesis.

Here, we have investigated the contribution of integrin-mediated adhesion to the ECM in the establishment and/or maintenance of cell polarity in vivo. To this end, we have analysed the consequences of integrin function removal on epithelial follicle cell polarity.

\section{Results}

\section{Polarization of epithelial follicle cells requires integrin-de- pendent and-independent mechanisms}

The Drosophila genome contains two integrin $\beta$ subunits, the $\beta P S$ subunit encoded by the myospheroidgene (mys) and the $\beta v$

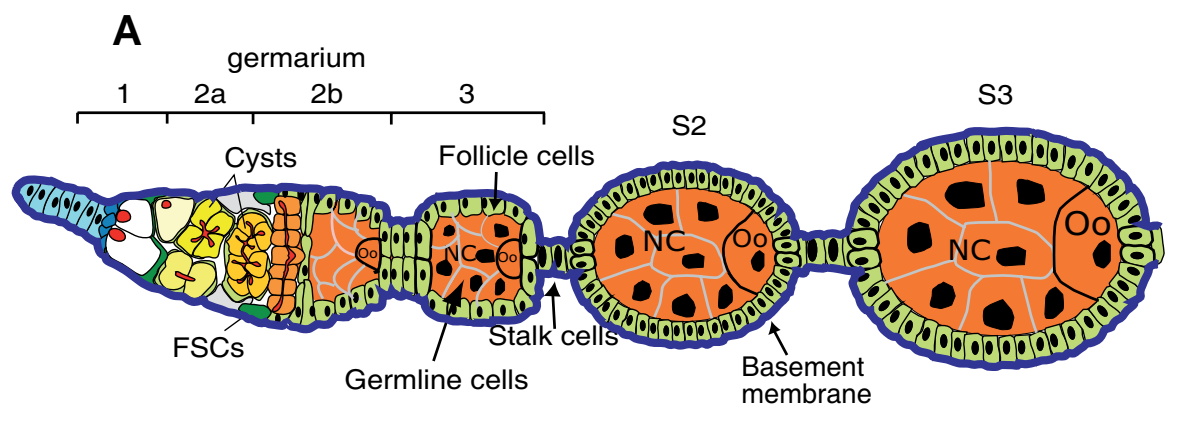

B
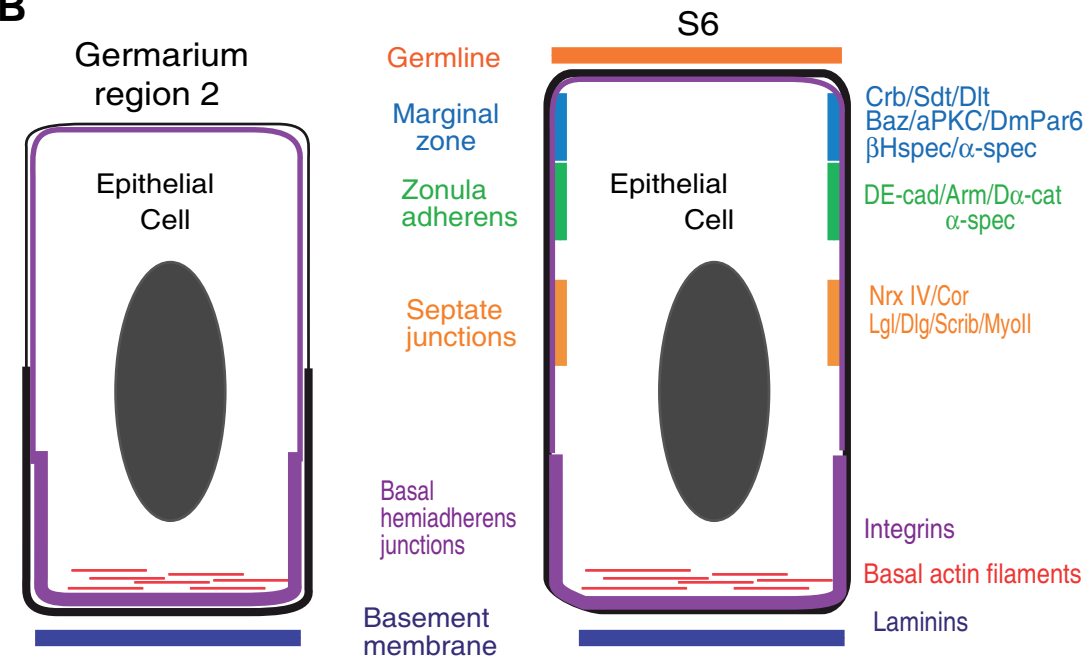

Fig. 1. Scheme of the acquisition of polarity of follicle cells. (A) Drawing of the anterior tip of an ovariole showing a germarium and stage 2 and 3 (S2 and S3) egg chambers. Follicle cells derive from two stem cells (FSCs) located at the boundary between regions $2 a / 2 b$. These stem cells give rise to both follicle cells that migrate to encapsulate the 16-cell germline cyst in region $2 b$ and to 6-8 stalk cells that form the interfollicular stalk. (B) Before contacting the germline, in region 2 of the germarium, follicle cells make contact with the basement membrane surrounding the ovariole. As a consequence, the basal domain of these cells is established. Around S6, contact with the germline leads to the establishment of apical and lateral domains within the follicle cell membrane. The distribution of proteins with a polarised localization is shown. Modified from Tanentzapfet al., 2000. 
chain (Brown, 2000). The $\beta$ PS subunit, which forms functional heterodimers with all five $\alpha$ subunits reported in the Drosophila genome, is the only $\beta$ chain present in the ovary (Brown, 2000, Devenport and Brown, 2004). We have recently shown that mosaic egg chambers containing follicle cell clones mutant for the null allele $m y s^{11}$ develop extra cell layers at both poles of the egg chamber and that integrin function was required to orientate the mitotic spindle parallel to the germ line (FernándezMiñán et al., 2007). We also noticed that although integrin mutant cells in contact with the germ line localize normally apical and lateral polarity markers, such as Bazooka (Baz) and Discs large (DIg), mutant cells in ectopic layers showed an aberrant distribution (Fernández-Miñán et al., 2007). In order to define in further detail the role of integrins in the polarisation of follicle cells, we decided to analyse the expression of different polarity markers such as Baz, DPatj, $\beta$ Heavy-spectrin ( $\beta_{\mathrm{H}}$-Spec), aPKC, $D \mathrm{E}$-Cadherin ( $D \mathrm{E}$-Cad) and Armadillo (Arm) in the follicular epithelium of egg chambers containing mys mutant follicle cells and that have developed extra layers. In those cases where a bi-layered epithelium is formed wild type and mutant cells are found to contact the germline or the basement membrane. As both the germline and the basement membrane provide cues for polarization, these bi-layered epithelia represent a good system to study the relative contributions of each polarization source. We have already demonstrated that mutant cells abutting the germline possess a normal apicalbasal polarity, a phenotype not affected by the simultaneous elimination of integrins from the germ line (Fernández-Miñán et al., 2007). Thus, we concentrated on follicle cells that having lost contact with the germline, maintain their connection with the basement membrane. Analysis of the localization of the polarity markers Baz and Dig in these mutant follicle epithelia revealed that mys mutant cells that loose contact with the germ line
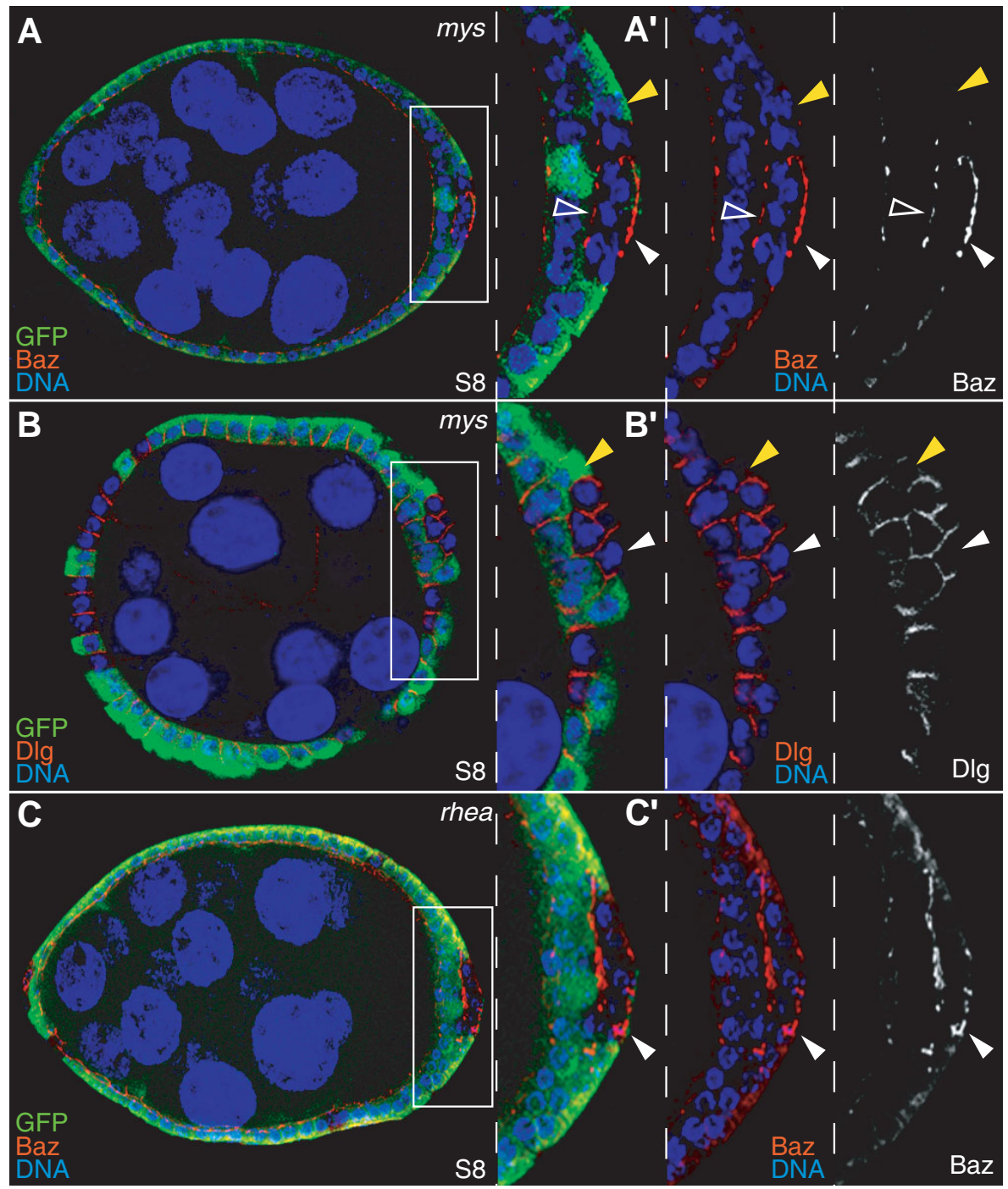

Fig. 2. Integrins are not required to maintain the apicobasal polarity of follicle cells in contact with the germ line. (A,B) Mosaic egg chambers carrying mys ${ }^{-}$germline clones and mys ${ }^{-}$follicle cell clones in the posterior terminal domain labelled with anti-GFP (green), TO-PRO-3 (blue) and with anti-Bazooka (Baz) (A) or anti-Discs large (D/g) (B) in red. Loss of contact with the germline results in abnormal distribution of Baz and Dlg in mys mutant cells (arrowheads in $\left(A^{\prime} B^{\prime}\right)$, respectively) while wild type cells in this situation do not show any polarity defects; see yellow arrowheads in ( $\left.\mathbf{A}^{\prime}, \mathbf{B}^{\prime}\right)$. Baz is also found in between the basal side of mys mutant cells in the ectopic layer and the apical side of cells in the inner layer (empty arrowhead). (C) An abnormal basal localization of Baz is also observed in rhea mutant follicle cells; see arrowhead in (C').

(arrowheads in Fig. 2A, B) showed an aberrant distribution of Baz and DIg. Thus, Baz was found both apical and basally and DIg was not longer found restricted to the lateral membranes. On the contrary, wild type cells that have lost contact with the germ line do not show any polarity defects (yellow arrowheads in Fig. 2A, B). These results strongly suggest that integrin-mediated cell-ECM interaction is required to polarize follicle cells when contact with the germ line is lost.

The analysis of the distribution of polarity markers in bi-layered epithelia showed an interesting localisation pattern of the apical marker Baz when mutant cells were present in the ectopic layer. Irrespective of the genotype of the follicle cell underneath, we observed that Baz was found in between the inner layer (in contact with the germline) and the ectopic layer (in contact with the basement membrane). This staining (empty arrowhead in Fig. 2A) could either correspond to the 'apical' side of cells in the ectopic layer or to the basal side of cells in the inner layer. If the former were true, it would indicate that the polarity of mys mutant cells in contact with the germ line is not affected, suggesting that contact with the germline is sufficient to induce proper polarization in the absence of integrins. Alternatively, if the mis-localisation of Baz corresponds even partially to the cell contacting the germ line, it would suggest that loss of integrin function in the ectopic layer could induce a non-autonomous polarity defect in the cells contacting the germline. Although at present we cannot distinguish between these two possibilities, we have previously demon- 
strated a local non-autonomy for integrin function in the follicular epithelium (Fernández-Miñán et al., 2007).

To further support our conclusion that integrins regulate cell polarity when contact with the germline is disrupted, we next analysed the polarity of follicle cells lacking Talin, a core component of the integrin complex encoded by rheain Drosophila(Brown etal., 2002). Analysis of the role of Talin during oogenesis revealed that the absence of Talin did not affect the normal distribution of polarity markers, such as Baz, aPKC, $\alpha$-spectrin or $\beta P S$ in follicle cells within the epithelium, suggesting that Talin was not required for the polarization of follicle cells (Becam et al., 2005). However, as we have found that removal of Talin gave rise to a stratification phenotype identical to that of loss of mys (Fernández-Miñán et al., 2007), we decided to analyse whether Talin was required to polarize the follicle cells in the extra layers. In agreement with an integrin-linked role for Talin in epithelial polarization, we found an aberrant distribution of Baz and DIg in rheamutant cells that are not in contact with the germline (arrowhead in Fig. 2C' and data not shown).

\section{A role for the germ line and the basement membrane in follicle cell polarity}

The above results suggest that contact with the basement membrane mediated by integrins is required for cell polarization when contact with germline cells is disrupted. However, in addition to its typical basolateral localization, $\beta$ PS integrins are found in the apicolateral domain in follicle cells. Thus, the polarity defects observed in integrin mutant cells may be due to a requirement for integrins in the apicolateral domain rather than to their more general role in mediating adhesion to the basement membrane. To directly address whether contact with the basement membrane has a role on cell polarity, we decided to analyse the polarity of wild type follicle cells that have lost contact with both the germline and the basement membrane. To this end, we analysed mosaic epithelia containing large clones of mys mutant follicle cells, as they can develop up to three or four extra layers that are composed of both mutant and wild type cells. As expected, wild-type cells that only contact the germline exhibited a normal distribution of Baz (empty arrowhead in Fig. 3A). Similarly, wild type cells that just contact the basement membrane also show normal distribution of apical markers, as Baz is only found on their apical surface facing the germ line (arrowhead in Fig. 3A). However, in wild-type cells in the internal layers, which neither adjoin the germ line nor the basement membrane (arrow in Fig. 3A), Baz is not found on the surface facing the germline. Staining with an antibody that recognizes the basement membrane component lamininA shows that these cells located in internal layers are not longer in contact with the laminin-rich basement membrane (arrow in Fig. 3B). Taken altogether, these results show that both the base-

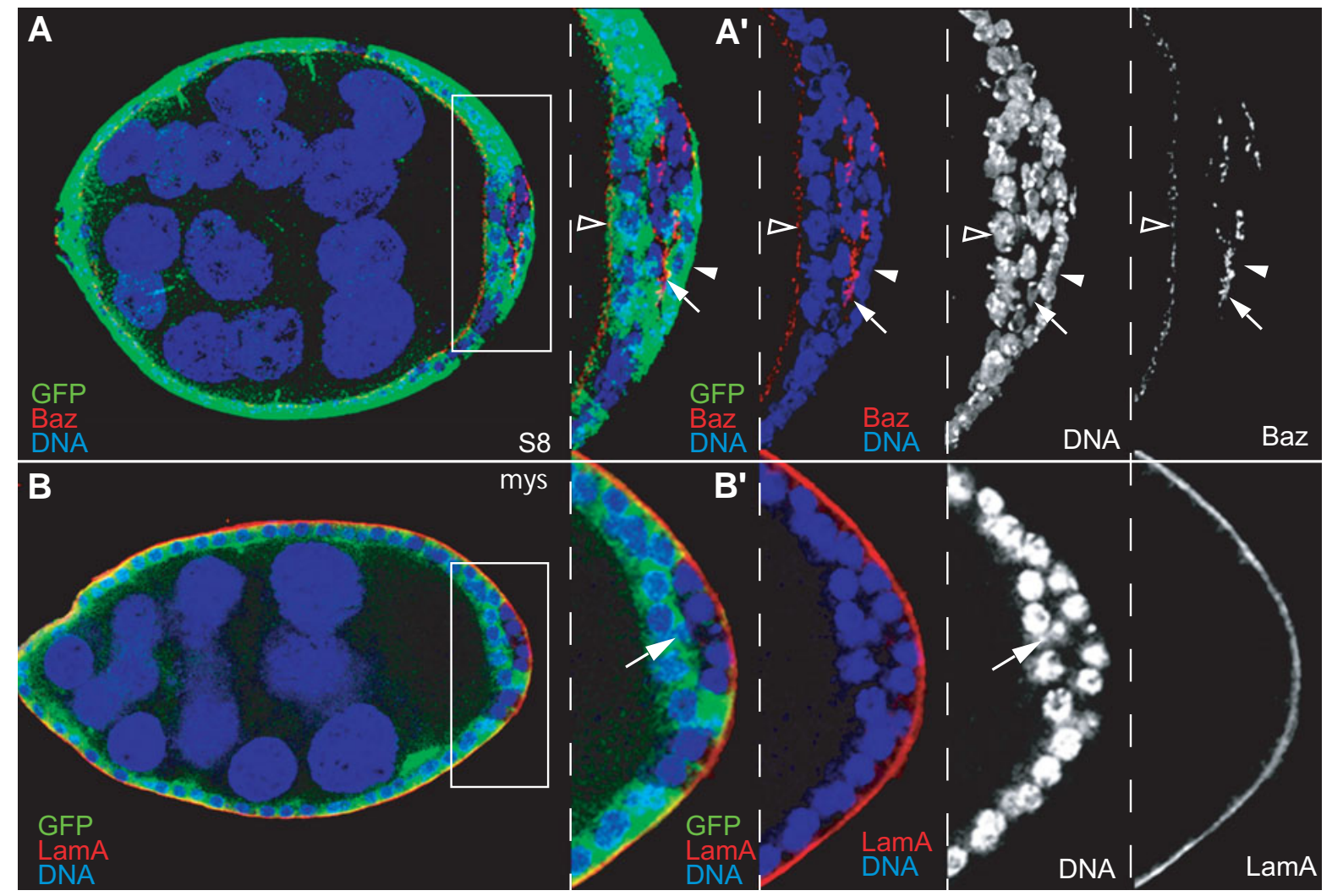

Fig. 3. Loss of contact with both the germ line and the basement membrane results in abnormal polarity. (A,B) Mosaic egg chambers harbouring mys mutant clones stained with anti-GFP (green), TO-PRO-3 (blue) and anti-Bazooka (A) or anti-Laminin A (B) (red). (A) Baz localizes apically in wild-type posterior follicle cells in contact with the germline (empty arrowhead). Similarly, wild-type cells in the most external layer display normal localization of Baz proximal to the oocyte (arrowhead). However, wild type cells (arrow) in intermediate layer do not show accumulation of Baz in their apical surfaces facing the oocyte. (B) Mosaic egg chamber displaying a stratified epithelium at the posterior pole stained with the ECM component Laminin A (red), anti-GFP (green) and TO-PRO3 (blue). The basement membrane, defined by the major concentration of Laminin A, is not found around the periphery of wild type follicle cells in the intermediate layers (arrow). 


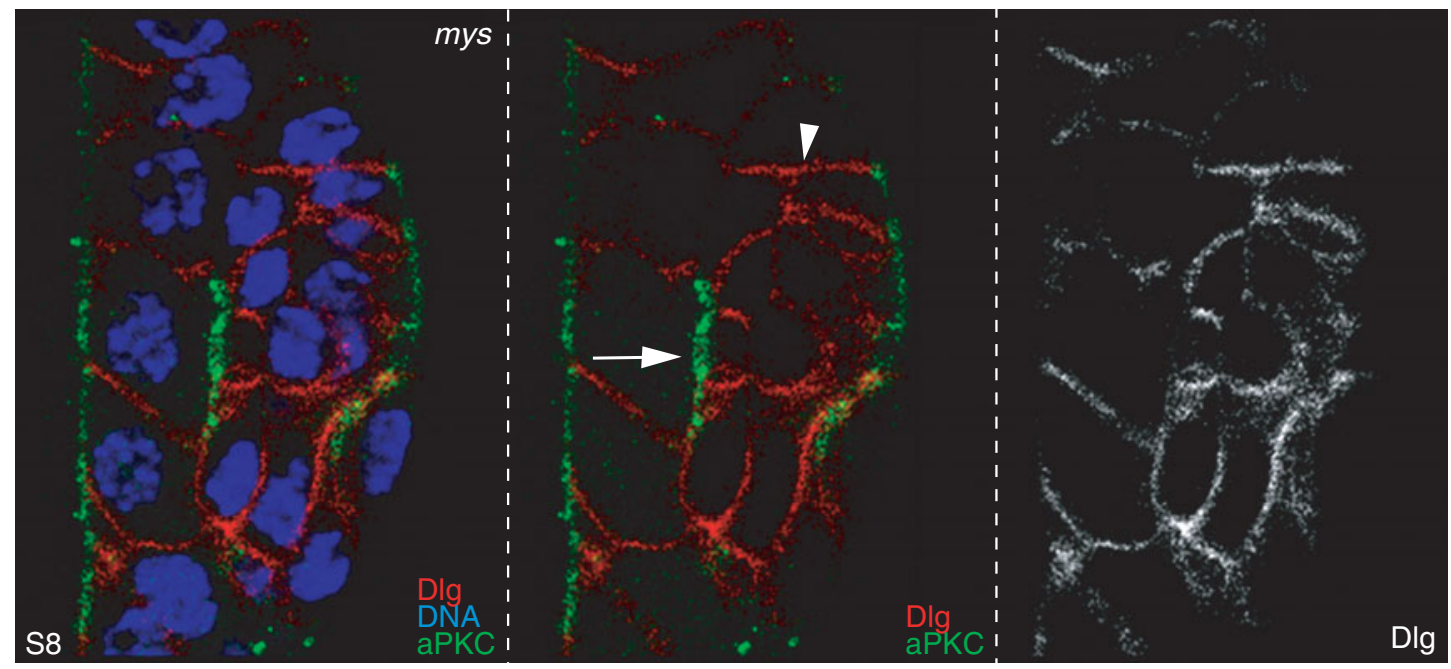

Fig. 4. Apical and lateral domains remain distinct in follicle cells lacking integrins. Posterior pole of a mosaic egg chamber containing mys mutant cells. The localization of the apical marker protein aPKC (green), lateral Dlg (red) and the DNA dye TO-PRO-3 (blue) is shown. In spite of the abnormal polarity of mutant cells within the ectopic layers, there is no co-localization of aPKC (arrow) and Dlg (arrowhead).

ment membrane and the germ line can on their own provide cues that are sufficient to induce proper follicle cell polarization. In addition, our results show that the role of the basement membrane on follicle cell polarity is integrin-dependent.

\section{Integrins are not required for differentiation of apicolateral domains}

As reported above, the distribution of Baz and Dlg in mys mutant cells depends on their position within the ectopic layers. Mutant cells adjoining the germ line localize polarity markers correctly. However, mutant cells belonging to the ectopic layers showed aberrant Baz and Dig distribution (Fig. 2A, B and Fig. 3A). Similar abnormal localization patterns were obtained for Patj, $D E$-Cad, Arm, $\beta_{H^{-}}$Spec, aPKC and $\alpha$-Spec (data not shown). This phenotype could reflect a problem in the proper differentiation of lateral and apical cell surface domains. In fact, a long-range effect of basal contact in the differentiation of apicolateral membrane domains has already been demonstrated in MDCK cells in culture. In this case, contact with the ECM, in the absence of cell-cell interactions and tight junctions, is sufficient to induce basal membrane domain formation and polarization of apical proteins (Vega-Salas et al., 1987). Thus, we decided to check whether apical and lateral domains are demarcated in follicle cells lacking integrins and that do not contact the germ line. We analysed the distribution of the apical marker aPKC and that of the lateral marker Dlg in mutant cells that are part of the ectopic layers. We found that mys mutant cells maintain the demarcation between apical and lateral membranes, as aPKC and DIg do not co-localize (Fig. 4). In addition, we noticed that in some mys mutant cells DIg and aPKC expanded over most of the cell surface (Fig. 4), suggesting that loss of integrin function might affect the proper differentiation of the basal membrane domain. Unfortunately, no markers are expressed exclusively on the basal domain of follicle cells that could allow us to directly assess a role for integrins on basal domain differentiation.

\section{Loss of $\beta P S$ integrin function in follicle cells does not induce apoptosis}

Loss of polarity as a result of interfering with apical or lateral cues may lead to cell death. For instance, follicle cells lacking the apical determinant $\mathrm{Crb}$ or the lateral adhesion molecule $D E$-Cadherin show an irregular morphology at early stages of oogenesis and degenerate at mid to late oogenesis (Tanentzapf et al., 2000). We have also observed that wild type and mys mutant cells that are not in contact with the basal lamina or the germ line lose their cuboidal shape and become smaller and rounded. In addition, it has been shown that complete loss of cell contact with the substrate mediated by integrins in cell culture results in programmed cell death (Frisch and Ruoslahti, 1997). Furthermore, changes in cell geometry due to integrin-loss of function seem to be crucial to regulate cell survival, as cells that are geometrically constrained undergo apoptosis (Singhvi et al., 1994). A role for integrins on the maintenance of apicobasal polarity and apoptosis has also been demonstrated in the development of the murine mammary gland. In this case, interfering with $\beta 1$ integrins function affects cell proliferation, maintenance of apicobasal polarity, apoptosis and differentiation of mammary epithelial cells (Faraldo et al., 1998a). Thus, we tested whether loss of integrin function in follicle cells would lead to apoptosis. To examine cell death we used an antiactive caspase-3 antibody (Yu et al., 2002). In wild type egg chambers, caspase-3 activity is only detected at high levels in randomly degenerating follicle cells, identified by their fragmented nuclei seen with the DNA marker TO-PRO3 (arrow in Fig. 5A). However, we did not find upregulation of caspase- 3 activity in any of the cells that compose the multilayer (Fig. 5B). These experiments suggest that integrins are not required to regulate cell survival within the follicular epithelium. This is in contrast with the function of another ECM receptor, Dystroglycan $(\mathrm{Dg})$, which in addition to its role on regulating apicobasal polarity in follicle cells, it is also required for their survival (Deng et al., 2003).

\section{Discussion}

The establishment and maintenance of epithelial polarity is essential to maintain proper homeostasis in the developing and adult organism, as it facilitates key cellular processes such as differentiation, localized membrane growth, vectorial transport of molecules across cell layers and regulation of cell-cell signalling events. Recently, the combined use of cell biological and genetic 

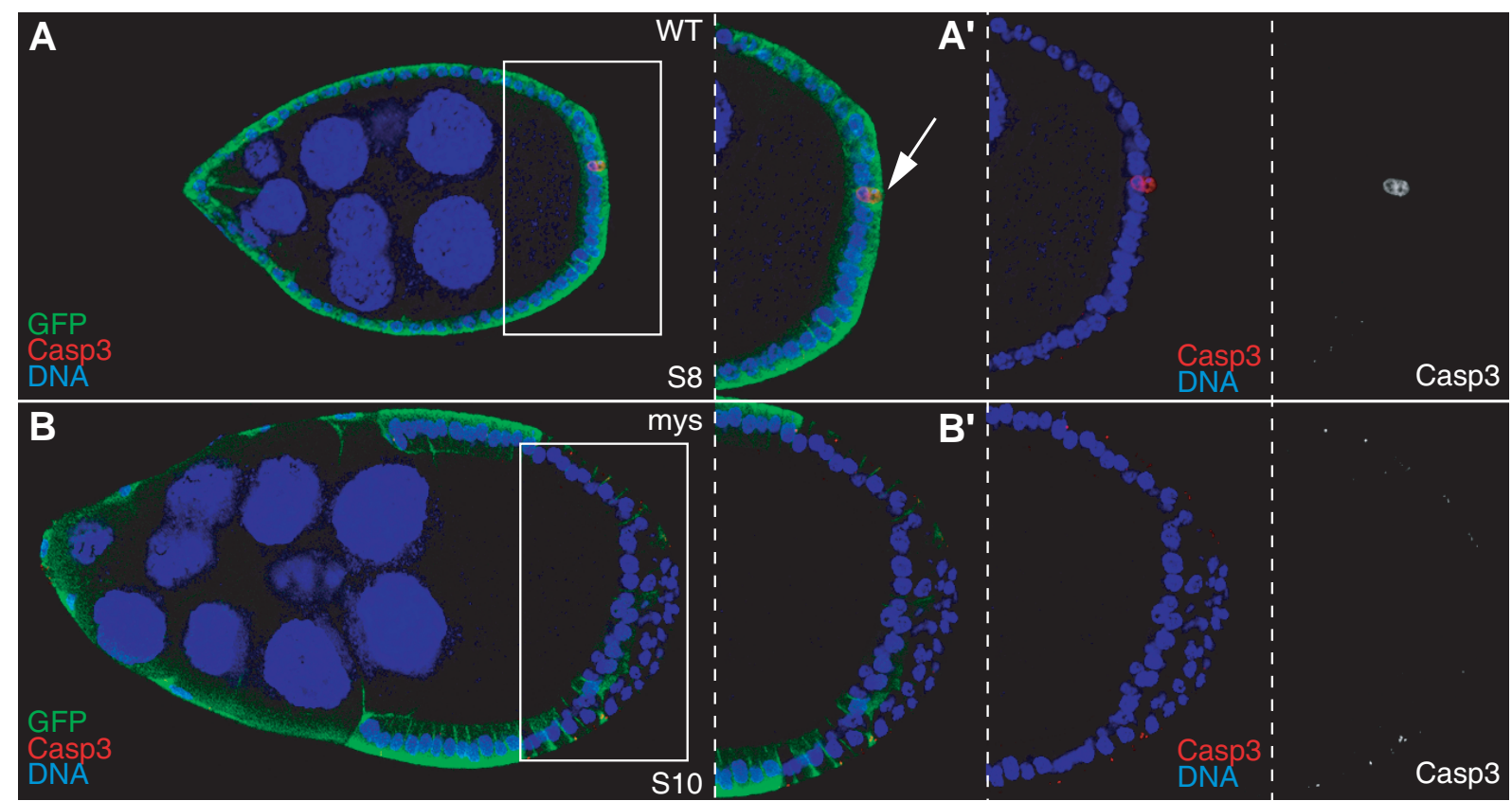

Fig. 5. Loss of integrin function in follicle cells does not lead to cell death. Wild type (A) and mosaic egg chambers containing mys follicle cell clones (B) labelled with anti-GFP (green), TO-PRO-3 (blue) and with anti-active caspase 3 (red). (A) In wild type egg chambers, high levels of active caspase 3 are only detected in seldom degenerating follicle cells; see arrow in ( $\left.A^{\prime}\right)$. (B) A similar situation is observed in the cells that compose the multilayer, irrespectively of whether they are wild type or mutant.

approaches in both cell culture and model organisms has revealed that the generation of cell polarity is a complex process that is beginning to be unravelled. For example, in spite of the amount of evidence showing that cell-cell interactions are crucial to generate cell polarity, recent experiments have demonstrated that intestinal epithelial cells can sort their apical and basolateral domains in the absence of cell-cell contact (Baas et al., 2004). Furthermore, as this cell-autonomous polarization is not randomly oriented, it is thought that these cells possess a prepatterned polarity that could have been set up by cell-ECM interactions. Here, we have addressed the contribution of the main cell-ECM receptors, the integrins, to the establishment of cell polarity in the developing follicular epithelium of the Drosophila ovary.

\section{Contribution of integrins to the establishment of apicobasal polarity}

Epithelial cell polarity in multicellular tissues is coupled to tissue architecture, thus ensuring the formation of a functional organ. This is achieved by the coordinated activity of different mechanisms operating at the apical, lateral or basal surfaces of epithelial cells. In the case of the Drosophila follicular epithelium, three mechanisms have been proposed to operate in parallel at the three cell surfaces (Tanentzapf et al., 2000). At the apical surface, contact with the germline cells is required for both the correct localization of apical determinants, such as Crb and DIt, and the demarcation of the lateral membrane. At the lateral surface, cell-cell adhesion mediated by the cadherin-catenin complex leads to the establishment of the lateral and apical surfaces. Finally, contact with the basement membrane causes partial polarization.

Our results show that interactions with the basement mem- brane via integrins are indeed required for proper polarization of follicle epithelial cells if contact with the germ line is lost. Integrins play a reinforcing role in this process, as they are required to establish or maintain follicle cell membrane asymmetry only when these cells do not abut the germ line. In addition, while interfering with apical and lateral polarization cues in the follicular epithelium results in eventual cell death, we have shown here that loss of contact with the basement membrane does not affect cell survival.Cell-ECM interactions have been shown to be critical for the establishment of cell polarity in cell culture (Nelson, 2003, O'Brien et al., 2002). The fact that integrin mutant epithelial cells still develop apicobasal polarity might reflect the complexity that the generation of polarity implies in developing multicellular tissues where numerous inputs operate. For example, follicle cells do not have a free apical surface, as cultured MDCK cells, but contact the germline cells. This most probably provide additional cues for polarization. In addition, follicle cells can use receptors other than integrins to contact the ECM or the basement membrane. In fact, Dystroglycan, another receptor for multiple ECM molecules is also required for epithelial polarity in follicle cells (Deng et al., 2003). Similarly to integrins, Dg mutant follicle cells form multilayers and show polarity defects. It would be interesting to analyse the consequences that removal of both ECM receptors have on follicle cell polarity.

\section{Integrins and the correct establishment of the basolateral domain}

Cell-ECM contact has also been involved in the resolution of tight junctions into discrete regions at the apex of the lateral membranes in developing MDCK cysts (Wang et al., 1990). Tight junctions are the functional equivalent in mammals to the Drosophila septate junctions (SJs), and are also important barriers that 
segregate apical and basolateral components (Bilder et al., 2003). Our results showing that the domain of expression of Dlg, a component of the septate junctions, is expanded in mys mutant follicle cells that do not adjoin the germ line support a role for integrins in the redistribution of SJs to discrete regions of the lateral membrane. Alternatively, the expansion of the lateral domain observed in mys mutant cells could be a consequence of a reduction of the basal membrane once contact with germline cells is prevented. This latter possibility is supported by our finding that in mys mutant cells apical and lateral markers decorate nearly the entire cell surface. A clear evidence for a role of integrins in specifying basal domain awaits the isolation of specific basal markers.

\section{Orientation of polarity}

Our studies show that mys mutant follicle cells in ectopic layers localize apical markers on the surface contacting the basement membrane, in contrast with the phenotype observed in mutants for genes essential for epithelial polarity, such as bazooka or discs large, in which apical markers are found around the entire cell surface (Abdelilah-Seyfried et al., 2003). This suggests that integrins might be required for proper orientation of cell polarity in absence of contact with the germ line. This is in agreement with the proposed role for $\beta 1$ integrins in MDCK cells. In suspension cultures, MDCK cells form polarized epithelial cysts with the apical surface facing the outside and the basolateral membrane lining the lumen. However, when these cysts are grown within collagen gels polarity reverses, a process that requires $\beta 1$ integrin function (Ojakian and Schwimmer, 1994, Wang et al., 1990). The mechanisms by which integrins regulate epithelial polarity in follicle cells remain unclear. In culture MDCK cysts, $\beta 1$ integrins lie upstream of Rac1 and Laminin to control the orientation of polarity (O'Brien et al., 2001, Yu et al., 2005). However, analysis in follicle cells of the role of one of the two laminins existing in Drosophila, $\operatorname{LanA}$, has revealed that the polarity of follicle cells is normal in lanA- mutant cells (Deng and Ruohola-Baker, 2000). In view of our results, it would be interesting to analyse whether removal of both laminins would have an effect on follicle cell polarity. Similarly, a role for Rac on follicle cell polarity needs to be investigated.

The correct functioning of epithelial tissues depends largely on the polarized organization of the individual cells within them. In addition, loss of cell polarity is a hallmark of cancer. Thus, a better understanding of the mechanisms that modulate biogenesis and maintenance of cell polarity in multicellular organisms is therefore fundamental to both developmental and cancer biology.

\section{Materials and Methods}

\section{Drosophila genetics}

To generate somatic and germline mutant clones we used the FRT/ FLP technique (Chou and Perrimon, 1992). Mutant clones were marked by the absence of GFP.

The following mutant alleles and chromosomes were used: $m y s^{11}$ (also known as $m y s^{X G 43}$ (Bunch etal., 1992); rhea ${ }^{79}$ (Brown etal., 2002) and e22c-Gal4 UAS- flp (Duffy et al., 1998). The e22c-Gal4 driver is expressed in the follicle stem cells in the germarium and was therefore used in combination with UAS- flp to generate large mys follicle cell clones. With a low frequency, the e22c-Gal4UAS- flp chromosome also induces recombination in the germ line.

\section{Immunohistochemistry and Microscopy}

Drosophila females were yeasted for 1-2 days before dissection. Stainings were performed at room temperature following standard procedures. Protocols are available upon request. The DNA dye TO-PRO-3 (Molecular Probes ${ }^{\mathrm{TM}} ; 1 / 1000$ ) was added for 10 minutes in PBT after the secondary antibody. The following primary antibodies were used: rabbit anti-GFP at 1/10000 (Molecular Probes ${ }^{\mathrm{TM}}$ ); mouse anti-GFP at $1 / 100$ (Molecular Probes ${ }^{\mathrm{TM}}$ ); rabbit anti-Bazooka at 1/500 (Wodarz et al., 1999); mouse anti-Discs Large at 1/100 (Developmental Studies Hybrodoma Bank, DSHB); mouse anti- $D E-C a d$ at 1/10 (DSHB); rabbit anti-aPKC at 1/10000 (PKC C20; Santa Cruz Biotechn. Inc.); rabbit anti-LamininA at 1/ 500 (Kumagai et al., 1997); rabbit anti-cleaved caspase 3 (Asp175) at 1/ 50 (Cell Signaling Technology). Secondary antibodies, FITC (Molecular Probes $^{\mathrm{TM}}$ ), and Cy3 (Jackson ImmunoResearch Laboratories, Inc.) were used at $1 / 200$. Images were captured with a Leica TCS-SP2 confocal microscope.

\section{ACKNOWLEDGMENTS}

We thank the Bloomington Stock Centre for fly stocks. Research in our laboratories is funded by the Spanish Ministerio de Ciencia y Tecnologia (MCYT) (BFU2006-10934 to A.G-R; BFU2004-02840/BMC to M.D.M-B), by the EMBO Young Investigator Programme and by the Junta de Andalucia (CVI-280 and PO6-CVI-01592). L. C-R was supported by a fellowship funded by the EMBO Young Investigator Programme and $A$. F$M$ was supported by a FPI studentship from the MCYT and by an I3PCSIC contract. The institutional support from the Junta de Andalucia to the $C A B D$ is acknowledged.

\section{References}

ABDELILAH-SEYFRIED, S., COX, D.N. and JAN, Y.N. (2003). Bazooka is a permissive factor for the invasive behavior of discs large tumor cells in Drosophila ovarian follicular epithelia. Development 130: 1927-1935.

BAAS, A., KUIPERS, J., VAN DER WEL, N.N., BATLLE, E., KOERTEN, H.K., PETERS, P.J. and CLEVERS, H.C. (2004). Complete polarization of single intestinal epithelial cells upon activation of LKB1 by STRAD. Ce//116: 457-466.

BATEMAN, J., REDDY, R., SAITO, H. and VAN VACTOR, D. (2001). The receptor tyrosine phsphatase Dlar and integrins organize actin filaments in the Drosophila follicular epithelium. Curr. Biol. 11: 1317-27.

BECAM, I.E., TANENTZAPF, G., LEPESANT, J.-A., BROWN, N.H. and HUYNH, J.R. (2005). Integrin-independent repression of cadherin transcription by talin during axis formation in Drosophila. Nat. Cell Biol. 7: 510-516.

BILDER, D., SCHOBER, M. and PERRIMON, N. (2003). Integrated activity of PDZ protein complexes regulates epithelial polarity. Nat. Cel/ Biol. 5: 53-58.

BROWN, N.H. (2000). Cell-cell adhesion via the ECM:integrin genetics in fly and worm. Matrix Biol. 19: 191-201.

BROWN, N.H., GREGORY, S.L., RICKOLL, W.L., FESSLER, L.I., PROUT, M., WHITE, R.A. and FRISTROM, J.W. (2002). Talin is essential for integrin function in Drosophila. Developmental Cel/3: 569-579.

BUNCH, T.A., SALATINO, R., ENGELSGJERD, M.C., MUKAI, L., WEST, R.F. and BROWER, D.L. (1992). Characterization of mutant alleles of myospheroid,the gene encoding the b subunit of the Drosophila PS integrins. Genetics 132: 519528.

CHOU, T.B. and PERRIMON, N. (1992). Use of a yeast site-specific recombinase to produce female germline chimeras in Drosophila. Genetics 131: 643-653.

DENG, W.-M. and RUOHOLA-BAKER, H. (2000). LamininA is required for follicle cell-oocyte signaling that leads to establishment of the anterior-posterior axis in Drosophila. Current Biology 10: 683-686.

DENG, W.-M., SCHNEIDER, M., FROCK, R., CASTILLEJO-LOPEZ, C., GAMAN, E.A., BAUMGARTNER, S. and RUOHOLA-BAKER, H. (2003). Dystroglycan is required for polarizing the epithelial cells and the oocyte in Drosophila. Deve/opment 130: 173-184. 


\section{A. Fernández-Miñán et al.}

DEVENPORT, D. and BROWN, N.H. (2004). Morphogenesis in the absence of integrins:mutation of both Drosophila $b$ subunits prevents midgut migration. Development 131: 5405-5415.

DUFFY, J.B., HARRISON, D.A. and PERRIMON, N. (1998). Identifying loci required for follicular patterning using directed mosaics. Developmen 125: 22632271.

FARALDO, M.M., DEUGNIER, A.A., LUKASHEV, M., THIERY, J.P. and GLUKHOVA, M.A. (1998a). Perturbation of b1-integrin function alters the development of murine mammary gland. EMBO J. 17: 2139-2147.

FARALDO, M.M., DEUGNIER, M.A., LUKASHEV, M., THIERY, J.P. and GLUKHOVA, M.A. (1998b). Perturbation of beta1-integrin function alters the development of murine mammary gland. EMBO J. 17: 2139-2147.

FERNÁNDEZ-MIÑÁN, A., MARTÍN-BERMUDO, M.D. and GONZÉALEZ-REYES, A. (2007). Integrin signaling regulates spindle orientation in Drosophila to preserve the follicular-epithelium monolayer. Current Biology 17: 683-688.

FRISCH, S.M. and RUOSLAHTI, E. (1997). Integrins and anoikis. Curr. Opin. Cell Biol. 9: 701-706.

KING, R.C. (1970). Ovarian development in Drosophila melanogaster. Academic Press, New York, NY.

KUMAGAI, C., KADOWAKI, T. and KITAGAWA, Y. (1997). Disulfide-bonding between Drosophila laminin beta and gamma chains is essential for alpha chain to form alpha betagamma trimer. FEBS Lett 412: 211-6.

MAHOWALD, A.P. (1972). Ultrastructural observations on oogenesis in Drosophila. J. Morphol. 137: 29-48.

MARGOLIS, B. and BORG, J.-P. (2005). Apicobasal polarity complexes. J. Cell Science 118: 5157-5159.

MARGOLIS, J. and SPRADLING, A. (1995). Identification and behaviour of epithelial stem cells in the Drosophila ovary. Development 121: 3797-3807.

MULLER, H.A. (2000). Genetic control of epithelial cell polarity: lessons from Drosophila. Developmental Dynamics 218: 52-67.

NELSON, W.J. (2003). Adaptation of core mechanims to generate cell polarity. Nature 422: 766-774.

O'BRIEN, L.E., JOU, T.-S., POLLACK, A.L., ZHANG, Q., HANSEN, S.H., YURCHENCO, P. and MOSTOV, K.E. (2001). Rac1 orientates epithelial apical polarity through effects on basolateral laminin assembly. Nature Cel/ Biology3: 831-838.

O'BRIEN, L.E., ZEGERS, M.M. and MOSTOV, K.E. (2002). Opinion: Building epithelial architecture:insights from three-dimensional culture models. Nat. Rev. Mol. Cell Biol. 3: 531-537.

OJAKIAN, G.K. and SCHWIMMER, R. (1994). Regulation of epithelial cell surface polarity reversal by b1 integrins. J. Cell Science 107: 561-576.

SINGHVI, R., KUMAR, A., LOPEZ, G.P., STEPHANOPOULOS, G.N., WANG, D.I., WHITESIDES, G.M. and INGBER, D.E. (1994). Engineering cell shape and function. Science 264: 696-698.

SOROKIN, L., SONNENBERG, A., AUMAILLEY, M., TIMPL, R. and EKBLOM, P. (1990). Recognition of the laminin E8 cell-binding site by an integrin possessing the alpha 6 subunit is essential for epithelial polarization in developing kidney tubules. J. Cell Biol. 111: 1265-1273.

SPRADLING, A.C. (1993). Developmental genetics of oogenesis. The Development of Drosophila melanogaster. (Eds. M. Bate and A Martinez-Arias). Cold Spring Harbor Lab. Press, Cold Spring Harbor, New York1-70.

TANENTZAPF, G., SMITH, C., MCGLADE, J. and TEPASS, U. (2000). Apical, Lateral, and Basal polarization cues contribute to the development of the follicular epithelium during drosophila oogenesis. J. Cell Biology 151: 891-904.

TEPASS, U., TANENTZAPF, G., WARD, R. and FEHON, R. (2001). Epithelal cell polarity and cell junctions in Drosophila. Annu. Rev. Genet. 35: 747-784.

VEGA-SALAS, D.E., SALAS, P.J.I., GUNDERSEN, D. and RODRIGUEZ-BOULAN, E. (1987). Formation of the apical pole of epithelial (Madin-Darby Canine Kidney)cells:polarity of an apical protein is independent of tight junctions while segregation of basolateral marker requires cell-cell interactions. J. Cell Biology 104: 905-916.

WANG, A.Z., OJAKIAN, G.K. and NELSON, W.J. (1990). Steps in the morphogenesis of a polarized epithelium. II. Disassembly and assembly of plasma membrane domains during reversal of epithelial cell polarity in multicellular epithelial (MDCK) cysts. J. Cell Science 95: 153-165.

WODARZ, A., RAMRATH, A., KUCHINKE, U. and KNUST, E. (1999). Bazooka provides an apical cue for Inscutable localization in Drosophila neuroblasts. Nature 402: 544-547.

YU, S.Y., YOO, S.J., YANG, L., ZAPATA, C., SRINIVASAN, A., HAY, B.A. and BAKER, N.E. (2002). A pathway of signals regulating effector and initiator caspases in the developing Drosophila eye. Development 129: 3269-3278.

YU, W., DATTA, A., LEROY, P., O'BRIEN, L.E., MAK, G., JOU, T.-S., MATLIN, K.S., MOSTOV, K.E. and ZEGERS, M.M.P. (2005). b1-integrins orients epithelial polarity via Rac1 and laminin. Molecular Biology of the Cel/16: 433-445. 


\section{Further Related Reading, published previously in the Int. J. Dev. Biol.}

See our recent Special Issue Fertilization, in honor of David L. Garbers and edited by Paul M. Wassarman and Victor D. Vacquier at: http://www.ijdb.ehu.es/web/contents.php?vol=52\&issue=5-6

See our recent Special Issue Ear Development edited by Fernando Giraldez and Bernd Fritzsch at:

http://www.ijdb.ehu.es/web/contents.php?vol=51\&issue=6-7

Acquisition of cell polarity during cell cycle and oral replacement in Tetrahymena

Janina Kaczanowska, Szymon Kaczanowski, Mauryla Kiersnowska, Hanna Fabczak, Karolina Tulodziecka and Andrzej Kaczanowski Int. J. Dev. Biol. (2008) 52: 249-258

Follicular cell differentiation in polytrophic ovaries of a moth midge, Tinearia alternata

Marta Mazurkiewicz and Janusz Kubrakiewicz

Int. J. Dev. Biol. (2008) 52: 267-278

Shaping the mammalian auditory sensory organ by the planar cell polarity pathway

Michael Kelly and Ping Chen

Int. J. Dev. Biol. (2007) 51: 535-547

Analysis of the role of the Rac/Cdc42 GTPases during planar cell polarity generation in Drosophila

Silvia Muñoz-Descalzo, Azucena Gómez-Cabrero, Marek Mlodzik and Nuria Paricio Int. J. Dev. Biol. (2007) 51: 379-388

Analysis of Netrin 1 receptors during inner ear development Tanja Matilainen, Maarja Haugas, Jordan A. Kreidberg and Marjo Salminen Int. J. Dev. Biol. (2007) 51: 409-414

Mechanical control of tissue morphogenesis during embryological development

Donald E. Ingber

Int. J. Dev. Biol. (2006) 50: 255-266

alphallb integrin, a novel marker for hemopoietic progenitor cells Catherine Corbel Pierre Vaigot and Josselyne Salaün

Int. J. Dev. Biol. (2005) 49: 279-284

Loss of beta1 integrin function results in upregulation of connexin expression in embryonic stem cell-derived cardiomyocytes

Jaroslaw Czyz, Kaomei Guan2, Qinghua Zeng3 and Anna M. Wobus

Int. J. Dev. Biol. (2005) 49: 33-41

2006 ISI ${ }^{* *}$ Impact Factor $=3.577^{* *}$

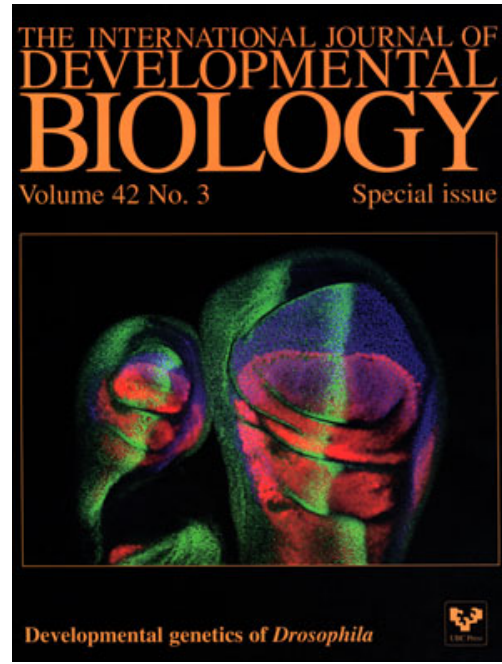

